\title{
Efficacy and Safety of Nintedanib for the Treatment of Idiopathic Pulmonary Fibrosis: An Update
}

\author{
José Antonio Rodríguez-Portal ${ }^{1,2}$
}

Published online: 5 December 2017

(c) The Author(s) 2017. This article is an open access publication

\begin{abstract}
Idiopathic pulmonary fibrosis is a fatal form of progressive fibrosing interstitial pneumonia with limited treatment options. In recent years, its management has been transformed with the approval of two new antifibrotic drugs: nintedanib and pirfenidone. Nintedanib is a tyrosine kinase inhibitor that efficiently slows idiopathic pulmonary fibrosis progression and has an acceptable tolerability profile. This article reviews new available evidence on the long-term efficacy and safety of nintedanib in patients with idiopathic pulmonary fibrosis. Data from extension trials indicate that nintedanib continues to slow disease progression for up to 3 years and is similarly effective in patients with mild and severe impairment of lung function. Treatment with nintedanib reduces the risk of acute exacerbations, and a combined analysis of data from clinical trials of nintedanib shows a trend towards a reduction in mortality. Nintedanib is well tolerated and has been shown to be safe for up to 51 months. Gastrointestinal events, mainly diarrhoea, are the main adverse events caused by the treatment. Currently available data confirm its safety profile in real-life clinical settings, with no new safety concerns identified in patients with comorbidities.
\end{abstract}

José Antonio Rodríguez-Portal jarportal@ecua.es

1 Centro de Investigación Biomédica en Red de Enfermedades Respiratorias (CIBERES), Madrid, Spain

2 Servicio de Neumología, Hospital Universitario Virgen del Rocío, Av. Manuel Siurot, s/n, 41013 Seville, Spain

\section{Key Points}

Idiopathic pulmonary fibrosis is a progressive form of fibrosing interstitial pneumonia with a fatal prognosis and a significant impact on the quality of life of affected patients.

Nintedanib was shown to be effective in reducing the decline in lung function of patients with idiopathic pulmonary fibrosis and was approved for treatment of idiopathic pulmonary fibrosis less than 3 years ago.

New studies indicate that nintedanib effectively slows disease progression up to 3 years, and is efficacious in mild, moderate and severe stages of the disease.

Nintedanib is well tolerated and safe up to 3 years, with diarrhoea being the most common adverse event.

\section{Introduction}

Idiopathic pulmonary fibrosis (IPF) is a fatal form of progressive fibrosing interstitial pneumonia. Advances in the understanding of its pathogenesis together with positive results from multiple clinical trials have transformed the management of IPF in the last decade. In recent years, two antifibrotic drugs have proved to be effective in slowing IPF progression: pirfenidone and nintedanib. Nintedanib 
was approved by the US Food and Drug Administration in October 2014 and by the European Medicines Agency in January 2015.

\subsection{Definition, Epidemiology and Diagnosis of Idiopathic Pulmonary Fibrosis}

Idiopathic pulmonary fibrosis is a specific form of chronic, progressive fibrosing interstitial pneumonia that occurs primarily in older male individuals. Idiopathic pulmonary fibrosis is a fatal disease, with a mean survival of 2-5 years from diagnosis $[1,2]$. Incidence estimates range from three to nine cases per 100,000 per year in Europe and North America [3] and prevalence is estimated to range between 2 and 29 per 100,000 in the general population $[1,2]$.

Clinical presentation of IPF includes unexplained progressive dyspnoea on exertion, usually associated with a non-productive cough, detection of inspiratory crackles on auscultation and finger clubbing in some cases (50\%). Diagnosis of IPF is complex and requires the exclusion of other interstitial lung diseases and the presence of a radiologic and/or histopathologic pattern of usual interstitial pneumonia. Accuracy of IPF diagnosis improves with a multidisciplinary discussion between pulmonologists, radiologists and pathologists experienced in the management of interstitial lung diseases $[1,2]$.

\subsection{Pathophysiology of Idiopathic Pulmonary Fibrosis}

The aetiology of IPF remains unknown; however, some risk factors have been associated with the disease, including cigarette smoking, gastroesophageal reflux, specific environmental exposures, viral infection and age. Similarly, some gene polymorphisms have been identified in patients with IPF $[1,2]$.

The current model of IPF pathogenesis proposes that chronic lung injury can trigger an aberrant tissue repair response in genetically susceptible individuals. Mediators released by damaged alveolar epithelial cells lead to excessive fibroblast proliferation and uncontrolled production of extracellular matrix components, which, eventually, cause fibrosis and loss of respiratory function [1, 2].

The natural history of IPF is variable and unpredictable at the time of diagnosis. Some patients experience a slow disease progression and others a rapid evolution; and some experience acute exacerbations: sudden deterioration episodes that occur during periods of relative stability of the disease $[1,2]$. Disease progression of IPF can be monitored with pulmonary function tests. Relevant measures for IPF are, among others, forced vital capacity (FVC) and diffusion capacity of lung for carbon monoxide. Declines in diffusion capacity of lung for carbon monoxide and FVC over time are used as a measure of disease progression and have prognostic value, especially FVC [2].

\subsection{TOMORROW and INPULSIS Trials}

Nintedanib is an orally available tyrosine kinase inhibitor originally developed to treat various types of cancer. Nintedanib inhibits the vascular endothelial growth factor receptors 1-3, platelet-derived growth factor receptors $\alpha$ and $\beta$, and fibroblast growth factor receptors $1-3$, which have been involved in the pathogenic process of IPF [4].

The first evidence of the efficacy of nintedanib in patients with IPF came from the TOMORROW trial, a multi-national, double-blinded, randomised, placebo-controlled, 52-week, phase II clinical trial [4]. In this study, patients were divided into five groups: placebo, nintedanib $50 \mathrm{mg}$ once a day, and nintedanib 50,100 and $150 \mathrm{mg}$ twice a day. Although being a phase II dose-finding study including multiple comparisons and adjustments for multiple testing, treatment with a $150-\mathrm{mg}$ dose of nintedanib twice a day showed a promising numerical trend towards a reduction in the annual rate of decline in FVC by approximately $68 \%$, from $0.19 \mathrm{~L} /$ year in the placebo group [95\% confidence interval (CI) $0.26-0.12$ ] to $0.06 \mathrm{~L} /$ year in the nintedanib group (95\% CI $0.14-0.02 ; p=0.06)$. The efficacy of nintedanib in slowing IPF progression was supported by a significant difference between groups in favour of nintedanib on the secondary endpoints of the trial. Nintedanib recipients had a reduction in the incidence of acute exacerbations and an improvement in their healthrelated quality of life [4].

The results from this study led to the design of the INPULSIS trials: two replicate, double-blind, randomised, placebo-controlled, 52-week, phase III clinical trials (INPULSIS-1 and INPULSIS-2) [5]. A total of 1061 patients from 24 countries participated in the INPULSIS trials. Consistent with the data from TOMORROW, nintedanib significantly reduced the annual rate of decline in FVC (pooled data; between-group difference of $109.9 \mathrm{~mL} /$ year; 95\% CI 75.9-144.0; $p<0.001$ ) [5]. A number of prespecified subgroup analyses of the INPULSIS trials showed that the beneficial effect of nintedanib is consistent irrespective of sex, age $(<65, \geq 65$ years) and race (White, Asian) and by FVC based on the $70 \%$ threshold $[6,7]$.

\section{Efficacy of Nintedanib}

After the completion of the INPULSIS trials, nintedanib was approved for IPF treatment in USA, Europe and Japan. In the latest update of the international clinical practice guideline for IPF management, nintedanib received a "conditional recommendation" with "moderate confidence 
in estimates of effect" [8]. The committee considered that future studies with nintedanib should address its long-term safety and efficacy, and include patients with comorbidities and more advanced disease (e.g. TOMORROW extension and INPULSIS-ON).

\subsection{Long-Term Efficacy of Nintedanib}

Comparator-controlled data of nintedanib for a period longer than 52 weeks come from an extension of the TOMORROW trial [9]. After completion of the 52-week period (period 1), patients could continue with nintedanib treatment in a further blinded period (period 2). Patients who were treated with $150 \mathrm{mg}$ twice a day in period 1 continued with the same treatment regimen, and patients from the placebo group switched to a $50 \mathrm{mg}$ once-daily nintedanib treatment (comparator group: placebo/nintedanib $50 \mathrm{mg}$ once daily). The effect of nintedanib on slowing IPF progression persisted up to week 76 (absolute change from baseline in FVC predicted; -3.1 and $-6.3 \%$ in patients in nintedanib and comparator groups, respectively) [10].

Descriptive data on the long-term efficacy of nintedanib come from INPULSIS-ON, an ongoing open-label extension trial where patients who completed the INPULSIS trials could continue with nintedanib treatment [11]. A total of 734 patients (out of 807 that completed the INPULSIS trials, $91 \%$ ) were treated in INPULSIS-ON (430 continuing nintedanib, 304 initiating nintedanib). An interim analysis of a data snapshot in October 2016 suggests that the efficacy of nintedanib in slowing respiratory function decline persists beyond 144 weeks [11]. Absolute declines in FVC in patients were measured from baseline to week 144. The mean total exposure for patients initiating nintedanib treatment in INPULSIS-ON was 28.8 months. The adjusted annual rates of decline in FVC of these patients during the 144-week period was similar to the decline in nintedanib recipients in the INPULSIS trials (INPULSIS-ON, weeks $0-144,-142.6$ and $-112.4 \mathrm{~mL} /$ year in patients who continued and initiated nintedanib, respectively; and INPULSIS, -113.6 vs. $-223.5 \mathrm{~mL} / \mathrm{year}$ in nintedanib and placebo groups, respectively). The mean total exposure for patients treated with nintedanib in both INPULSIS and INPULSIS-ON was 40.7 months, with a maximum exposure of 63.1 months. Together, these results suggest that nintedanib is efficacious in reducing the decline in FVC at least up to 3 years [11].

\subsection{Nintedanib is Efficacious Irrespective of the Stage of Disease}

A number of pre-specified and post hoc subgroup analyses of INPULSIS trials have tested the effect of baseline
FVC $\%$ predicted on the efficacy of nintedanib (FVC \% predicted $\leq 70$ vs. $>70, \leq 80$ vs. $>80$ and $\leq 90$ vs. $>90 \%$ ) and the annual rate of decline in FVC was similar in all nintedanib recipients, irrespective of FVC impairment at baseline $[6,7,12]$. This indicates that treatment with nintedanib could be used not only in patients with a mild-tomoderate impairment of lung function, but also in patients with more preserved lung volume (FVC $>90 \%)$ [7]. In addition, a post hoc subgroup analysis of both INPULSIS trials showed that nintedanib slowed disease progression independent of the presence of emphysema at baseline [13]. Thus, nintedanib is effective in a broad spectrum of patients.

Results regarding the efficacy of nintedanib in patients with severe impairment of lung function $(\mathrm{FVC} \leq 50 \%)$ have only recently become available [14], as one of the inclusion criteria in TOMORROW and INPULSIS trials was an FVC $\%$ predicted $>50 \%[4,5]$. In contrast, patients from the INPULSIS trials who had experienced a decline to an FVC $\leq 50 \%$ could continue with nintedanib treatment in the INPULSIS-ON trial [11], and the efficacy of nintedanib in advanced disease could be evaluated [11]. Absolute decline in FVC over 48 weeks was measured in 24 patients with baseline FVC $\leq 50 \%$. The absolute decline in FVC was similar in patients with a severe impairment of respiratory function and in patients with mild-to-moderate impairment of respiratory function [absolute mean (standard error of the mean [SEM])] change in FVC, $-62.3 \mathrm{~mL}$ (63.13) vs. $-87.9 \mathrm{~mL}(10.0)$, in patients with baseline FVC $\leq 50 \%$ and $>50 \%$ predicted, respectively] [14]. Limitations of this study include potential population bias owing to the open-label extension design, and the small number of participants in the $\mathrm{FVC} \leq 50 \%$ subgroup. However, these are the first results that show a beneficial effect of an antifibrotic agent on patients with IPF with baseline FVC $\leq 50 \%$ [14]. This suggests that patients with severely impaired lung function may also benefit from treatment with nintedanib.

The efficacy of nintedanib has been also demonstrated in a real-world clinical setting. Prior to the approval and launch of nintedanib in Germany, patients with IPF who were not eligible for pirfenidone treatment could be included in a compassionate use programme of nintedanib [15]. Compared with the participants of INPULSIS trials, patients in this programme were older [age, mean (SEM); 71 (8) vs. 66.8 (8.0) years], had a lower baseline FVC [FVC \% predicted, mean (SEM); 64 (17\%) vs. 79.6\% $(17.8 \%)]$ and had been diagnosed with IPF for a longer time [time after diagnosis, mean (SEM); 5.8 (2.4) vs. 1.6 (1.3) years] $[6,15]$. Patients included in the study had a mean FVC decline of $-7.4 \%$ within the previous 6 months of inclusion. Consistent with data from the TOMORROW and INPULSIS trials, nintedanib reduced disease 
progression in the majority of the patients. After 6 months of nintedanib treatment, $63 \%$ of patients $(n=30 / 48)$ had a FVC decline smaller than 5\% from baseline, and thus were considered to have stable disease [15].

The authors of the study noted that nintedanib treatment stabilised disease progression in $62 \%(n=13 / 21)$ of the patients who had not responded to pirfenidone in the previous 6 months [15]. In these patients, the mean (SEM) FVC change in the 6 months prior to nintedanib was $-17.3 \%(6 \%)$. After 6 months of nintedanib treatment, the mean (SEM) FVC change of the responders was $2 \%$ $(1.8 \%)$. In the remaining patients $(n=8 / 21)$, IPF continued to progress [FVC \% change, mean (SEM), $-15.7 \%$ (4\%) before nintedanib treatment compared with $-18 \%$ $(11 \%)$ after treatment] [15]. Although further studies are needed, these observations suggest that nintedanib may be an effective treatment option in patients who are not eligible for pirfenidone treatment or who did not respond to treatment with pirfenidone.

\subsection{Nintedanib Reduces Acute Exacerbations}

Acute exacerbations of IPF are defined as episodes of respiratory worsening that occurs within 1 month and cannot be explained by a known associated condition such as pneumonia, pulmonary embolism, pneumothorax or cardiac failure $[2,16]$. Acute exacerbations are associated with significant morbidity and an increase in mortality; therefore, time to the first acute exacerbation is considered a clinically relevant measure [16]. TOMORROW and INPULSIS trials yielded mixed results regarding the effect of nintedanib on acute exacerbations. In INPULSIS-1, patients from nintedanib and placebo groups did not differ in the time to the first acute exacerbation [hazard ratio (HR) 1.15 ; 95\% CI $0.54-2.42 ; p=0.67$ [ [4]. In contrast, nintedanib significantly increased the time to the first acute exacerbation in INPULSIS-2 (HR 0.38; 95\% CI 0.19-0.77; $p=0.005$ ), and reduced the incidence of acute exacerbations in TOMORROW (2.4 vs. 15.7 per 100 patient-years, $p=0.02)[3,4]$.

A recent study combined all the data from patients treated with nintedanib $150 \mathrm{mg}$ in the TOMORROW and INPULSIS trials [17]. The pooled analysis of data from the three trials showed that a smaller percentage of patients in the nintedanib group experienced acute exacerbations in a 52 -week period compared with patients in the placebo group (4.6 vs. 8.7\%) [17]. Treatment with nintedanib reduced by $47 \%$ the risk of experiencing a first investigator-reported acute exacerbation (HR $0.53 ; 95 \%$ CI $0.34-0.83 ; p=0.0047$ ) [17]. A beneficial effect of nintedanib vs. placebo was also observed when specifically examining the acute exacerbations reported as serious adverse events (HR 0.57; 95\% CI 0.32-0.99; $p=0.0476$ )
[18]. Acute exacerbations reported as serious adverse events occurred in $3.6 \%$ patients in the nintedanib group vs. $6.1 \%$ in the placebo group [18]. Finally, results in favour of nintedanib are more pronounced in patients with greater lung function impairment because they present with more acute exacerbations [6].

Consistent with the results from TOMORROW and INPULSIS trials, data from the INPULSIS-ON trial suggest that nintedanib is efficacious in reducing the number of acute exacerbations at least up to 3 years [11]. The adjusted incidence of acute exacerbations in patients of INPULSIS-ON at 144 weeks (5.3 and 5.2 per 100 patientyears, in patients continuing and initiating nintedanib treatment, respectively) was similar to the incidence observed in the nintedanib recipients in INPULSIS trials (5.2 per 100 patient-years); and smaller than the incidence of acute exacerbations in placebo recipients $(8.0$ per 100 patient-years) [11].

Mortality is the most clinically significant outcome of IPF. Pooled analysis of data from TOMORROW and INPULSIS trials showed that nintedanib reduces the risk of on-treatment mortality by $43 \%$ (HR $0.57 ; 95 \%$ CI $0.34-0.97 ; p=0.0274)$ [17]. Although non-statistically significant, similar trends in favour of nintedanib were detected for all-cause mortality (8.3 vs. 5.8\%) [HR 0.70; 95\% CI $0.46-1.08 ; p=0.0954]$ and respiratory mortality (5.7 vs. $3.6 \%$ ) [HR $0.62 ; 95 \%$ CI $0.37-1.06 ; p=0.0779$ ] [17].

Another important measure associated with mortality is the decline of lung function. An absolute reduction of more than $10 \%$ in FVC predicted (or significant decline in FVC) over 6 months is associated with increased mortality (HR 2.80; 95\% CI 1.5-5.06; $p<0.001$ ) [19]. Smaller declines in FVC are also clinically relevant: an absolute reduction of $5-10 \%$ in FVC predicted over 6 months is associated with increased mortality (HR 2.31; 95\% CI 1.19-4.50; $p=0.01$ ) [19]. A recent post hoc analysis of pooled INPULSIS data examined the cumulative distribution of patients by change in FVC over 52 weeks [20]. Compared with the patients in the placebo group, a greater percentage of patients in the nintedanib group had no decline or an improvement in FVC (30 vs. $15 \%$ in nintedanib and placebo groups, respectively) [odds ratio (OR) $2.5 ; 95 \% \mathrm{CI}$ $1.9-3.5 ; p<0.001]$. Conversely, a smaller percentage of nintedanib recipients had a significant decline in FVC (FVC decline $\geq 10 \%$ ) compared with placebo recipients (30 vs. $39 \%$ in nintedanib and placebo groups, respectively) [OR 1.6; 95\% CI 1.2-2.1; $p<0.001$ ]. Similarly, a smaller percentage of patients in the nintedanib group experienced a marginal decline in FVC (FVC decline $\geq 5 \%$ ) [47 vs. $61 \%$ in nintedanib and placebo groups, respectively] (OR 1.8; 95\% CI 1.4-2.4; $p<0.001$ ) [20]. 
Thus, nintedanib reduces the number of patients that experience both significant and marginal declines in FVC, and the incidence of acute exacerbations. Overall, this supports the effect of nintedanib in slowing disease progression, and suggests that nintedanib treatment may ultimately reduce mortality in patients with IPF.

\section{Safety of Nintedanib}

Nintedanib has a tolerability profile that is acceptable and manageable for the majority of patients, with most adverse events being of mild-to-moderate severity. The most frequent adverse events of nintedanib treatment are gastrointestinal events, mostly diarrhoea. Pooled analysis of data from TOMORROW and INPULSIS trials showed that $61.5 \%$ of patients in the nintedanib group reported diarrhoea, vs. $17.9 \%$ in the placebo group [17]. Premature treatment discontinuation because of adverse events occurred in $20.6 \%$ of patients in the nintedanib group vs. $15.0 \%$ of patients in the placebo group. Diarrhoea was the most frequent cause of premature discontinuation in nintedanib recipients, accounting for 5.3\% of the cases [17].

The occurrence of severe and serious adverse events did not differ between groups (26.7 vs. $23.4 \%$ of severe adverse events in nintedanib vs. placebo groups, respectively; and 30.0 vs. $30.1 \%$ of serious adverse events in nintedanib vs. placebo groups, respectively) [17]. An imbalance in myocardial infarction events was observed in disfavour of patients treated with nintedanib in the INPULSIS trials ( $n=10$ vs. $n=2$ patients in nintedanib and placebo groups, respectively). However, serious adverse events reflecting ischemic heart disease as a broader concept were balanced ( $n=15$ vs. $n=10$ patients in nintedanib and placebo groups, respectively) [5]. Other adverse events that should be taken into account include bleeding and liver enzyme elevation. Vascular endothelial growth factor receptor inhibitors may increase the risk of bleeding, and bleeding events were observed in INPULSIS trials (10 vs. $7 \%$ in nintedanib and placebo groups, respectively) [21]. Regarding hepatic function, $14 \%$ of patients treated with nintedanib presented elevated liver enzymes vs. $3 \%$ of patients in the placebo group [21]. Elevation of liver enzymes after nintedanib was reversible after dose reduction, treatment interruption or treatment termination; however, liver function tests are recommended before nintedanib prescription and periodically during treatment [21]. In general, management of adverse effects includes dose reduction (from 150 to $100 \mathrm{mg}$ twice a day) or treatment interruption [21], as well as symptomatic treatment in the case of diarrhoea (e.g. loperamide) $[1,22]$.
Data from INPULSIS-ON confirm the tolerability and safety profiles of nintedanib [11]. The incidence of diarrhoea adverse events was lower in patients of INPULSISON (62.5 and 73.7 per 100 patient-years in patients continuing and initiating nintedanib treatment, respectively) than in patients in the nintedanib group of INPULSIS trials (112.6 per 100-patient years) [11]. Treatment discontinuation because of diarrhoea adverse events observed in INPULSIS-ON (4.4 and 10.5 per 100 patient-years in patients continuing and initiating nintedanib treatment, respectively) was comparable to that of the INPULSIS trials (4.4 per 100 patient-years) [11]. These data from INPULSIS-ON confirm that nintedanib treatment had an acceptable safety and tolerability profile at least up to 63 months [11].

The tolerability and safety of nintedanib have also been evaluated in real-life clinical settings, such as the compassionate use programme study in Germany and a postmarketing surveillance in USA [15, 23]. Incidences of adverse events in real-life clinical settings are consistent with the findings of clinical trials, with gastrointestinal events (i.e. diarrhoea, nausea and vomiting) being the most frequently reported $[15,23]$ in a large post-marketing surveillance study in USA where data from 6758 patients treated with nintedanib were collected 1 year after product launch [23]. The median duration of exposure to nintedanib was 113 days (range 6-390 days). Of the 4062 cases of adverse events of special interest, 322 cases had a fatal outcome, including 27 cases of major adverse cardiac events, eight cases of myocardial infarction, three cases of stroke and two cases of bleeding.

There was no increment in the incidence of major adverse cardiac events ( 2.9 vs. 3.9 per 100 patient-years for nintedanib recipients in post-marketing surveillance and INPULSIS, respectively) and myocardial infarctions (1.0 vs. 1.7 per 100 patient-years) in nintedanib recipients of this study compared with the INPULSIS trials [23]. The rate of bleeding events was also similar to the rate reported in the INPULSIS trials. The majority of bleeding events with nintedanib were non-serious, with the most frequently reported events being epistaxis and contusions [23]. No bleeding events were reported in the compassionate use programme [15]. In conclusion, data from clinical real-life settings confirm the tolerability and safety of nintedanib because no new safety concerns were identified.

\section{Discussion and Conclusion}

Since the completion of the INPULSIS trials and the approval of nintedanib for IPF treatment, new data on its efficacy and safety are available. Extended trials have allowed us to understand better the long-term effects and 
safety of nintedanib with data up to 63 months [11]. The efficacy is maintained during the time reported so far, with no new adverse events reported.

Additionally, various subgroup analyses have shown that nintedanib is efficacious in a large variety of patients independently of age, sex and stage of the disease $[6,11,14]$. Interestingly, nintedanib would be beneficial from patients with marginal and advanced disease, including slowing the rate of IPF progression and reducing the rate of acute exacerbations. In addition, nintedanib is beneficial to patients with IPF with concomitant emphysema.

We also have relevant information from the real-world setting, with information on the effectiveness and safety of nintedanib in a broader spectrum of patients [15]. Data in the real-world setting suggest beneficial results in patients with a more severe disease and aged older than the population in the INPULSIS and TOMORROW trials.

Ongoing clinical trials will help to evaluate the efficacy and safety of nintedanib and pirfenidone in combination [1]. In this regard, it has been proposed that the combination of nintedanib and pirfenidone could present additional benefits to patients with IPF. One phase II study in Japanese patients has compared monotherapy with nintedanib vs. nintedanib add-on to long-term treatment with pirfenidone for a maximum treatment period of 28 days [24]. Data from this study indicated that the bioavailability of nintedanib might be lower when co-administered with pirfenidone; however, inter-subject variability was moderate to high. A small increase in adverse effects in patients receiving combined therapy vs. patients receiving monotherapy was also observed [24]. Another open-label randomised trial of nintedanib with add-on pirfenidone, compared with nintedanib alone for a period of 12 weeks, in patients with IPF (INJOURNEY trial, NCT02579603) showed that treatment add-on pirfenidone had a manageable safety and tolerability profile in patients with IPF, in line with the adverse profiles of the individual drugs [25]. The trough PK data obtained in this trial did not replicate the results of the phase II trial [23], in which the nintedanib plasma concentration tended to be lower following administration with pirfenidone. Finally, a smaller numerical decline in FVC over 12 weeks was observed in patients treated with nintedanib with add-on pirfenidone than with nintedanib alone [25]. Further studies are required to confirm these results and support further research into combination regimens in the treatment of IPF.

Regardless of the lack of a head-to-head comparison, it is conceptually clear that the treatment should start as early as possible to preserve pulmonary function and prolong survival (except, possibly, in those patients with asymptomatic disease or with marginal lung function impairment) [26]. In general, the decision of choosing one drug over the other is driven by the side-effect profile of each drug [27]. Furthermore, the convenience of treatment in relation to treatment satisfaction and adherence has to be taken into account considering that pill burden is higher in pirfenidone than in nintedanib.

In summary, nintedanib treatment has been shown to be safe at least up to 51.1 months. The most frequent adverse event was diarrhoea, which is manageable in most cases with symptomatic/anti-diarrheal therapies. A small percentage of patients discontinued the treatment because of this event [11]. Moreover, the use of nintedanib in real-life clinical settings shows no new safety concerns [15, 23]. All of this evidence provides support for nintedanib being efficacious, well tolerated and safe for up to 3 years for the treatment of IPF in mild, moderate and severe stages of the disease.

Acknowledgements The author thanks the medical writers from Adelphi Spain for their help with writing the manuscript.

\section{Compliance with Ethical Standards}

Funding Medical writing assistance, supported financially by Boehringer Ingelheim, was provided by Adelphi Spain during the preparation of this manuscript. Boehringer Ingelheim was given the opportunity to check the data used in this manuscript for factual accuracy only.

Conflict of interest The author is a member of Boehringer Ingelheim and Roche Scientific Advisory Board, Investigator in Clinical Trials and receives lecture fees from Boehringer Ingelheim and Roche.

Open Access This article is distributed under the terms of the Creative Commons Attribution-NonCommercial 4.0 International License (http://creativecommons.org/licenses/by-nc/4.0/), which permits any noncommercial use, distribution, and reproduction in any medium, provided you give appropriate credit to the original author(s) and the source, provide a link to the Creative Commons license, and indicate if changes were made.

\section{References}

1. Richeldi L, Collard HR, Jones MG. Idiopathic pulmonary fibrosis. Lancet. 2017;389:1941-52.

2. Raghu G, Collard HR, Egan JJ, Martinez FJ, Behr J, Brown KK, et al. An official ATS/ERS/JRS/ALAT statement: idiopathic pulmonary fibrosis: evidence-based guidelines for diagnosis and management. Am J Respir Crit Care Med. 2011;183:788-824.

3. Hutchinson J, Fogarty A, Hubbard R, McKeever T. Global incidence and mortality of idiopathic pulmonary fibrosis: a systematic review. Eur Respir J. 2015;46:795-806.

4. Richeldi L, Costabel U, Selman M, Kim DS, Hansell DM, Nicholson AG, et al. Efficacy of a tyrosine kinase inhibitor in idiopathic pulmonary fibrosis. N Engl J Med. 2011;365:1079-87.

5. Richeldi L, du Bois RM, Raghu G, Azuma A, Brown KK, Costabel U, et al. Efficacy and safety of nintedanib in idiopathic pulmonary fibrosis. N Engl J Med. 2014;370:2071-82.

6. Costabel U, Inoue Y, Richeldi L, Collard HR, Tschoepe I, Stowasser S, et al. Efficacy of nintedanib in idiopathic pulmonary 
fibrosis across prespecified subgroups in INPULSIS. Am J Respir Crit Care Med. 2016;193:178-85.

7. Kolb M, Richeldi L, Behr J, Maher TM, Tang W, Stowasser S, et al. Nintedanib in patients with idiopathic pulmonary fibrosis and preserved lung volume. Thorax. 2017;72:340-6.

8. Raghu G, Rochwerg B, Zhang Y, Garcia CAC, Azuma A, Behr J, et al. An official ATS/ERS/JRS/ALAT clinical practice guideline: treatment of idiopathic pulmonary fibrosis. Am J Respir Crit Care Med. 2015;192:e3-19.

9. Richeldi L, Costabel U, Selman M, Xu Z, Kimura T, Stowasser S, Hallmann C, Du Bois R. Efficacy and safety of nintedanib in patients with IPF beyond week 52: data from the phase II TOMORROW trial. Am J Respir Crit Care Med. 2015;191:A1019.

10. Richeldi L, Kreuter M, Selman M, Crestani B, Kirsten A-M, Wuyts WA, et al. Long-term treatment of patients with idiopathic pulmonary fibrosis with nintedanib: results from the TOMORROW trial and its open-label extension. Thorax. 2017;183:788-824.

11. Crestani B, Quaresma M, Kaye M, Stansen W, Stowasser S, Kreuter M. Long-term nintedanib treatment in idiopathic pulmonary fibrosis (IPF): new data from INPULSIS ${ }^{\circledR}$ [ON P1710317 P17-10317]. In: the European Respiratory Society International Congress. Milan, 9-13 September 2017; pp 1-11.

12. Maher T, Flaherty K, Noble P, Vancheri C, Wuyts W, Kimura T, et al. Effect of baseline FVC on lung function decline with nintedanib in patients with IPF. In: The European Respiratory Society International Congress. Amsterdam, 26-30 September 2015. 46 (suppl 59).

13. Pfeifer M, Cottin V, Taniguchi H, Richeldi L, Collard H, Kaye $\mathrm{M}$, et al. Effect of baseline emphysema on reduction in FVC decline with nintedanib in the INPULSIS ${ }^{\mathrm{TM}}$ trials. Pneumologie. 2015;69:P254.

14. Wuyts WA, Kolb M, Stowasser S, Stansen W, Huggins JT, Raghu G. First data on efficacy and safety of nintedanib in patients with idiopathic pulmonary fibrosis and forced vital capacity of $\leq 50 \%$ of predicted value. Lung. 2016;194(5):739-43.

15. Bonella F, Kreuter M, Hagmeyer L, Neurohr C, Keller C, Kohlhaeufl MJ, et al. Insights from the German compassionate use program of nintedanib for the treatment of idiopathic pulmonary fibrosis. Respiration. 2016;92:98-106.

16. Song JW, Hong S-B, Lim C-M, Koh Y, Kim DS. Acute exacerbation of idiopathic pulmonary fibrosis: incidence, risk factors and outcome. Eur Respir J. 2011;37:356-63.

17. Richeldi L, Cottin V, du Bois RM, Selman M, Kimura T, Bailes $Z$, et al. Nintedanib in patients with idiopathic pulmonary fibrosis: combined evidence from the TOMORROW and INPULSIS $^{\circledR}$ trials. Respir Med. 2016;113:74-9.

18. Kreuter M, Koegler H, Trampisch M, Geier S, Richeldi L. Efficacy of nintedanib on acute exacerbations reported as serious adverse events in the INPULSIS ${ }^{\circledR}$ trials in idiopathic pulmonary fibrosis (IPF) [P16- 05633]. American Thoracic Society Meeting. San Francisco, 13-18 May 2016; 193:A2690.

19. Zappala CJ, Latsi PI, Nicholson AG, Colby TV, Cramer D, Renzoni EA, et al. Marginal decline in forced vital capacity is associated with a poor outcome in idiopathic pulmonary fibrosis. Eur Respir J. 2010;35:830-6.

20. Flaherty KR, Brown KK, Costabel U, Stansen W, Schlenkerherceg R, Raghu G. Cumulative distribution of patients by change in FVC $\%$ predicted in the INPULSIS ${ }^{\circledR}$ trials of nintedanib in patients with idiopathic pulmonary fibrosis [P16-05631]. American Thoracic Society Meeting. San Francisco, 13-18 May 2016;193:A2705.

21. European Medicines Agency. Ofev (nintedanib) soft capsules: EU summary of product characteristics. 2015. http://www.ema. europa.eu/docs/en_GB/document_library/EPAR_-_Product Information/human/003821/WC500182474.pdf. Accessed 01 Dec 2017.

22. Xaubet A, Molina-Molina M, Acosta O, Bollo E, Castillo D, Fernández-Fabrellas E, et al. Normativa sobre el tratamiento farmacológico de la fibrosis pulmonar idiopática. Arch. Bronconeumol. 2017;53:263-9.

23. Noth I, Oelberg D, Allinger A, Kaul M, Conoscenti CS. Safety and tolerability of nintedanib in patients with idiopathic pulmonary fibrosis (IPF): data from post-marketing surveillance in the United States. American Thoracic Society Meeting. San Francisco, 13-18 May 2016; 193:A2692.

24. Ogura T, Taniguchi H, Azuma A, Inoue Y, Kondoh Y, Hasegawa Y, et al. Safety and pharmacokinetics of nintedanib and pirfenidone in idiopathic pulmonary fibrosis. Eur Respir J. 2015;45:1382-92.

25. Vancheri C, Kreuter M, Richeldi L, Ryerson CJ, Valeyre D, Grutters JC, et al. Nintedanib with add-on pirfenidone in idiopathic pulmonary fibrosis: results of the INJOURNEY trial. Am J Respir Crit Care Med. 2017; https://doi.org/10.1164/rccm. 201706-1301OC (Epub ahead of print).

26. Kreuter M, Bonella F, Wijsenbeek M, Maher TM, Spagnolo P. Pharmacological treatment of idiopathic pulmonary fibrosis: current approaches, unsolved issues, and future perspectives. Biomed Res Int. 2015;2015:329481.

27. Rangarajan S, Locy ML, Luckhardt TR, Thannickal VJ. Targeted therapy for idiopathic pulmonary fibrosis: where to now? Drugs. 2016;76:291-300 